\title{
MODELAGEM NÃO LINEAR DA RELAÇÃO HIPSOMÉTRICA E DO CRESCIMENTO DAS ÁRVORES DOMINANTES E CODOMINANTES DE Eucalyptus sp.
}

\author{
MODELING OF NONLINEAR HYPSOMETRIC RELATION AND GROWTH OF \\ DOMINANT AND CODOMINANT TREES OF Eucalyptus sp.
}

\author{
Elliezer de Almeida Melo ${ }^{1}$ Natalino Calegario ${ }^{2}$ Adriano Ribeiro de Mendonça ${ }^{3}$ \\ Ernani Lopes Possato ${ }^{4}$ Joyce de Almeida Alves ${ }^{5}$ Marcos Antonio Isaac Júnior ${ }^{5}$
}

\begin{abstract}
RESUMO
Este trabalho teve por objetivo avaliar modelos lineares e não lineares clássicos e generalizados com adição de covariáveis, para modelagem da relação hipsométrica e do crescimento em altura das árvores dominantes e codominantes de clones de eucalipto. Foram ajustados dois modelos lineares e dois não lineares para estimativa da relação hipsométrica e quatro modelos não lineares para classificação de sítio. Com relação à hipsometria, para o modelo que propiciou as melhores estatísticas, utilizou-se a técnica de inclusão de covariáveis para o ajuste, visando obter melhor precisão das estimativas. A seleção e a qualidade de ajuste dos modelos se deram com base no erro padrão percentual " $\mathrm{S}_{\mathrm{yx}}(\%)$ ", critério de informação de Akaike (AIC), critério de informação Bayesiano (BIC) e no teste de razão da máxima verossimilhança (TRMV), além da análise gráfica dos resíduos. Para a manipulação, ajuste e processamento dos dados foi utilizado o software R. De acordo com as análises estatísticas dos modelos, para a relação hipsométrica, o logístico com quatro parâmetros mostrou-se superior em relação aos outros modelos ajustados. O modelo logístico com adição de covariáveis obteve uma melhor precisão em comparação ao modelo logístico clássico. Para a estimativa do crescimento em altura das árvores dominantes e codominantes, o modelo logístico com três parâmetros obteve as melhores estatísticas de precisão, sendo, então, utilizado para a construção das curvas de índice de sítio.
\end{abstract}

Palavras-chave: eucalipto; modelos de regressão; modelos generalizados; covariáveis.

\section{ABSTRACT}

This work aimed to evaluate linear and nonlinear, classical and generalized models with the addition of covariates for modeling the hypsometric relation and the height growth of dominant and codominant trees of eucalypt clones. Two linear models and two nonlinear models were fitted to estimate the hypsometric relationship and four nonlinear models to classify the site. Regarding the hypsometry, it was used the technique of inclusion of covariates in the model that provided the best statistics in order to obtain more precise estimates. The selection and quality of the fittings was based on the standard error " $\mathrm{S}_{\mathrm{yx}}(\%)$ ", Akaike

1 Engenheiro Florestal, Doutorando em Ciências Florestais, Universidade Federal de Lavras, Professor Instituto Federal Goiano, Campus Morrinhos, BR-153, km 633, CEP 75650-000, Morrinhos (GO), Brasil. elliezer.melo@ifgoiano.edu.br

2 Engenheiro Florestal, PhD., Professor do Departamento de Ciências Florestais, Universidade Federal de Lavras, Caixa Postal 3037, CEP 37200-000, Lavras (MG), Brasil. calegari@dcf.ufla.br

3 Engenheiro Florestal, Dr., Professor do Departamento de Ciências Florestais e da Madeira, Universidade Federal do Espírito Santo, Av. Governador Lindemberg, 316, Bairro Centro, CEP 29550-000, Jerônimo Monteiro (ES), Brasil. adriano.mendonca@ufes.br

4 Engenheiro Florestal, Dr., Instituto de Ciências Agrárias-Engenharia Florestal, Universidade Federal de Uberlândia, Campus Monte Carmelo-Unidade Araras, MG-746, km 01, CEP 38500-000, Monte Carmelo (MG), Brasil.epossato@yahoo.com.br

5 Engenheiro(a) Florestal, Doutorando(a) em Ciências Florestais, Universidade Federal de Lavras, Caixa Postal 3037, CEP 37200-000, Lavras (MG), Brasil. joyce_a.alves@yahoo.com.br / maisaacjr@yahoo.com.br

Recebido para publicação em 30/01/2014 e aceito em 28/04/2016 
information criterion (AIC), Bayesian information criterion (BIC) and the test of maximum likelihood (LRATIO), in addition to the graphical analysis of the residuals. For handling, fitting and processing the data, it was used software ' $\mathrm{R}$ '. According to the statistical analysis of the models, for the hypsometric relation the four parameters logistic model was proved to be superior when compared to the other adjusted models. The logistic model obtained better accuracy compared to the classical model. In relation to the estimates of height growth in dominant and codominant trees, the three parameter logistic model obtained the best statistical precision, and therefore it was used for the construction of site index curves.

Keywords: eucalypt; regression models; generalized models; covariates.

\section{INTRODUÇÃO}

Conhecer a produtividade de um povoamento florestal é um requisito que o manejador deve atentar em toda parte do ciclo do povoamento, pois possibilita maior controle do planejamento, culminando em decisões eficazes e sólidas do empreendimento florestal. O uso de modelos de regressão para obtenção de informações do povoamento florestal é uma técnica válida, pois, se utilizada de maneira adequada, fornece estimativas de grande confiabilidade de variáveis de difícil obtenção no inventário florestal. Com os avanços tecnológicos, o aprimoramento de computadores e desenvolvimento de hardwares e softwares cada vez mais modernos, proporcionou uma evolução no uso de modelos florestais. $\mathrm{O}$ grande avanço ocorreu no processo de estimar uma variável do povoamento a partir de modelos não lineares. Além do uso de modelos não lineares, outras técnicas envolvendo a manipulação destes modelos foram desenvolvidas. A adição de variáveis do povoamento que explicam a variabilidade do que se deseja conhecer é um aprimoramento da modelagem. Essa técnica, também chamada de modelagem com adição de covariáveis, é utilizada com o intuito de produzir estimativas com alto grau de precisão (CALEGARIO et al., 2005).

Juntamente com as técnicas de modelagem faz-se necessário que o profissional saiba onde utilizálas. Daí vem à necessidade de conhecer as variáveis que afetam o crescimento e a produção florestal. Quando se deseja quantificar o volume de árvores individuais é necessário o conhecimento da altura total da árvore $\left(\mathrm{H}_{\mathrm{t}}\right)$, associada ao diâmetro a 1,30 metros de altura (DAP). Nesse contexto, pensando em economia e redução de erros no processo de obtenção dessas informações, aproximadamente $20 \%$ dos indivíduos em cada unidade amostral é mensurada a $\mathrm{H}_{\mathrm{t}}$, sendo o DAP, mensurado $100 \%$ em todas as unidades amostrais. Considerando o custo e as dificuldades encontradas na medição da $\mathrm{H}_{\mathrm{t}}$, tem-se a necessidade de utilizar as técnicas de modelagem para estimativa dessa variável. Além do DAP, outras variáveis podem ser inseridas no modelo, pois explicam a variabilidade $\mathrm{da}_{\mathrm{t}}$, como a idade, a capacidade produtiva do local, entre outras. Entre os trabalhos que seguem essa tendência têm-se: Machado et al. (1994), Bartoszeck et al. (2004a; 2004b), Temesgen e Gadow (2004), Tomé, Ribeiro e Faias (2007) e Mendonça et al. (2011).

O índice de sítio também é uma variável utilizada em estudos de crescimento e produção. Este corresponde à altura média das árvores dominantes e codominantes em uma idade de referência $\left(\overline{\mathrm{HDC}}_{\mathrm{ref}}\right)$. A altura dominante e codominante é a variável mais utilizada para classificação da capacidade produtiva, para determinada espécie ou clone, e para um determinando produto (CAMPOS; LEITE, 2009).

A relação entre a altura dominante e codominante e a idade vem sendo utilizada há muito tempo para estudos de classificação da qualidade do local e para compor modelos de crescimento e produção. Dentre os vários trabalhos pode-se citar: Amateis e Burkhart (1985), Corral Rivas et al. (2004), DiéguezAranda, Burkhart e Rodríguez-Soalleiro (2005), Tonini, Schneider e Finger (2006), Mendonça et al. (2011) e Kitikidou, Petrou e Milios (2012).

O objetivo do estudo foi avaliar modelos lineares e não lineares para modelagem da relação hipsométrica e do crescimento em altura das árvores dominantes e codominantes de povoamentos de clones de eucalipto. 


\section{MATERIAL E MÉTODOS}

\section{Caracterização da área de estudo e coleta dos dados}

Os dados para a realização do estudo foram provenientes de um plantio clonal de eucalipto, pertencente à empresa Copebrás, de propriedade do grupo Anglo American, localizada no município de Catalão e Ouvidor, região sudeste do estado de Goiás, Brasil. O clima da região enquadra-se no tipo Aw, que, de acordo com a classificação climática de Köppen, apresenta dois períodos distintos: um chuvoso e outro seco. Com temperatura média anual atingindo $22^{\circ} \mathrm{C}$ e precipitação média anual com valores entre 1200 e $1800 \mathrm{~mm}$.

A área total utilizada para o estudo foi de aproximadamente 1000 hectares. O método de amostragem foi o delineamento de amostragem casual simples (ACS). As unidades amostrais utilizadas possuíam formato retangular de área variável com aproximadamente $450 \mathrm{~m}^{2}$. Para o estudo da relação hipsométrica

TABELA 1: Frequências de diâmetro e altura das árvores-amostra presentes nas unidades amostrais.

TABLE 1: Diameter frequencies and height of sample trees present in the sample units.

\begin{tabular}{lccccccc}
\hline \multirow{2}{*}{ Classe DAP } & \multicolumn{6}{c}{ Classe de Altura Total } & \multirow{2}{*}{ Total } \\
\cline { 2 - 6 } 5,5 & 9,5 & 14,5 & 19,5 & 24,5 & 29,5 & 34,5 & 21 \\
8,5 & 15 & 6 & & & & & 68 \\
11,5 & 6 & 32 & 30 & & & & 228 \\
14,5 & 7 & 72 & 77 & 68 & 4 & & 562 \\
17,5 & & 32 & 106 & 300 & 122 & 2 & 842 \\
20,5 & & 3 & 22 & 299 & 478 & 40 & 289 \\
23,5 & & & & 35 & 190 & 64 & 99 \\
26,5 & & & & 1 & 69 & 29 & 33 \\
29,5 & & & & & 21 & 12 & 3 \\
Total & 28 & 145 & 235 & 703 & 885 & 149 & 2145 \\
\hline
\end{tabular}

TABELA 2: Estatísticas descritivas relacionadas às variáveis do povoamento.

TABLE 2: Descriptive statistics related to the stand variables.

\begin{tabular}{|c|c|c|c|c|c|}
\hline Variável do Povoamento & Mínimo & Máximo & Média & Mediana & $\mathrm{CV}(\%)$ \\
\hline Idade (anos) & 1,70 & 6,90 & - & - & 25,63 \\
\hline $\mathrm{N}$ & 891 & 1612 & 1189 & 1191 & 7,15 \\
\hline $\mathrm{S}$ & 27,00 & 39,00 & 33,86 & 33,00 & 6,46 \\
\hline$\overline{\mathrm{Dq}}$ & 10,16 & 18,53 & 15,50 & 15,72 & 8,95 \\
\hline $\mathrm{G}$ & 10,18 & 34,74 & 22,58 & 22,99 & 17,37 \\
\hline
\end{tabular}

Em que: $\mathrm{N}=$ número de árvores por hectare $\left(\mathrm{N} \mathrm{ha}^{-1}\right) ; \mathrm{S}=$ índice de sítio $(\mathrm{m}) ; \overline{\mathrm{Dq}}=$ diâmetro quadrático $(\mathrm{cm})$; $\mathrm{G}=$ área basal por hectare $\left(\mathrm{m}^{2} \mathrm{ha}^{-1}\right)$. 
e construção de curvas de índice de sítio, foram utilizados dados de 35 parcelas temporárias e 55 parcelas permanentes medidas em 2010 e 2012, distribuídas em diferentes idades e locais. Com base nos dados coletados, DAP e $\mathrm{H}_{t}$, procedeu-se a divisão das árvores em classes de diâmetro e de altura (Tabela 1), além de uma análise descritiva relacionada às variáveis do povoamento (Tabela 2).

\section{Ajuste de modelos hipsométricos e modelos para classificação da capacidade produtiva}

Foram ajustados modelos lineares e não lineares para a relação hipsométrica, sendo que, para a estimativa da altura média das árvores dominantes e codominantes ( $\overline{\mathrm{HDC}}$ ), apenas modelos não lineares. Para a manipulação dos dados, ajuste dos modelos e análises estatísticas foi utilizado o software R.

\section{Relação hipsométrica}

Para o ajuste da relação hipsométrica foram utilizados os modelos lineares e os modelos não lineares conforme Tabela 3.

Segundo Calegario et al. (2005), o modelo logístico apresenta maior facilidade de interpretação dos parâmetros. O modelo logístico de três parâmetros é composto pela assíntota horizontal, ponto de inflexão, e ponto de escala. A assíntota refere-se ao crescimento médio máximo, dado pelo eixo Y. O ponto de inflexão expressa a taxa média de máximo crescimento, dado pelo eixo X. O parâmetro de escala representa a distância do eixo $X$ entre o ponto de inflexão e, aproximadamente, $73 \%$ do máximo crescimento médio (PINHEIRO; BATES, 2000).

O modelo logístico de quatro parâmetros é composto pela assíntota horizontal superior e inferior, ponto de inflexão e ponto de escala. A assíntota horizontal superior e inferior $\left(\beta_{0}\right.$ e $\left.\beta_{1}\right)$, expressa a taxa média de máximo e mínimo crescimento, respectivamente, dado pelo eixo Y. O parâmetro $\beta 2$ representa o ponto de inflexão, dado pelo eixo X. O parâmetro de escala representa a distância do eixo X, aproximadamente, $75 \%$ da distância entre o parâmetro e (PINHEIRO; BATES, 2000).

Além da utilização de modelos não lineares para produção de estimativas mais precisas, o uso de

TABELA 3: Modelos para a estimativa da altura total de árvores de eucalipto.

TABLE 3: Models to estimate the total height of eucalypt trees

\begin{tabular}{|c|c|}
\hline \multicolumn{2}{|c|}{ Modelos Lineares } \\
\hline Modelo & Função Hipsométrica \\
\hline Curtis & $\operatorname{Ln}\left(H_{t}\right)_{i}=\beta_{0}+\beta_{1} \frac{1}{D P_{i}}+\varepsilon_{i}$ \\
\hline Parabólico & $\mathrm{H}_{\mathrm{t}_{\mathrm{i}}}=\beta_{0}+\beta_{1} \mathrm{DAP}_{\mathrm{i}}+\beta_{2} \mathrm{DAP}_{\mathrm{i}}^{2}+\varepsilon_{\mathrm{i}}$ \\
\hline \multicolumn{2}{|c|}{ Modelos Não Lineares } \\
\hline
\end{tabular}

Logístico 3 Parâmetros

$$
\mathrm{H}_{\mathrm{t}_{\mathrm{i}}}=\frac{\beta_{0}}{1+\exp \left[\left(\beta_{1}-\mathrm{DAP}_{\mathrm{i}}\right) / \beta_{2}\right]}+\varepsilon_{\mathrm{i}}
$$

Logístico 4 Parâmetros

$$
\mathrm{H}_{\mathrm{t}_{\mathrm{i}}}=\beta_{0}+\frac{\beta_{1}-\beta_{0}}{1+\exp \left[\left(\beta_{2}-\mathrm{DAP} \mathrm{P}_{\mathrm{i}}\right) / \beta_{3}\right]}+\varepsilon_{\mathrm{i}}
$$

Em que: $\mathrm{H}_{\mathrm{t}^{1}}=$ altura total da i-ésima árvore $(\mathrm{m}) ; \mathrm{DAP}_{\mathrm{i}}=$ diâmetro a $1,30 \mathrm{~m}$ de altura $(\mathrm{cm})$ da i-ésima árvore presente em cada unidade amostral; $\ln =$ logaritmo neperiano; $\exp =$ base do logaritmo neperiano; $\varepsilon$ 's = parâmetros a serem estimados; $\varepsilon_{\mathrm{i}}=$ erro aleatório. 
técnicas avançadas de modelagem deve ser uma prática corrente no meio florestal. Tal fato é justificado pela ineficiência de apenas uma variável explicar o que se deseja estimar. Nesse caso, para estimativa do crescimento em altura total de árvores individuais, os parâmetros do melhor modelo encontrado serão decompostos para inclusão de variáveis do povoamento que influenciam no crescimento. A idade (I), a capacidade produtiva do local (S) e a área basal $(\mathrm{G})$ foram as covariáveis inseridas no modelo.

\section{Classificação da capacidade produtiva}

Para classificação da capacidade produtiva foram testados quatro modelos não lineares. $\mathrm{O}$ uso de modelos não lineares para a classificação da capacidade produtiva se justifica pela capacidade de interpretação de seus parâmetros e pelo comportamento não linear da relação $\overline{\mathrm{HDC}}$ e idade.

$\mathrm{Na}$ Tabela 4 podem ser visualizados os modelos ajustados para classificação da capacidade produtiva de clones de Eucalyptus sp. da empresa Copebrás.

TABELA 4: Modelos não lineares ajustados para estimativa da altura média das árvores dominantes e codominantes, e classificação da capacidade produtiva.

TABLE 4: Adjusted models to estimate the average height of dominant and codominant trees and productive capacity classification.

\begin{tabular}{cc}
\hline Modelo & Função \\
\hline
\end{tabular}

Schumacher

$$
\overline{\mathrm{HDC}}_{\mathrm{i}}=\beta_{0} \exp \left(-\beta_{1} \frac{1}{\mathrm{I}_{\mathrm{i}}}\right)+\varepsilon_{\mathrm{i}}
$$

Chapman e Richards

$$
\overline{\mathrm{HDC}}_{\mathrm{i}}=\beta_{0}\left[1-\exp \left(-\beta_{1} \mathrm{I}_{\mathrm{i}}\right)\right]^{\beta_{2}}+\varepsilon_{\mathrm{i}}
$$

Bailey e Clutter 3 Parâmetros

$$
\overline{\mathrm{HDC}}_{\mathrm{i}}=\beta_{0}\left[1-\exp \left(-\beta_{1} \mathrm{I}_{\mathrm{i}} \beta_{2}\right)\right]+\varepsilon_{\mathrm{i}}
$$

Logístico 3 Parâmetros

$$
\overline{\mathrm{HDC}}_{\mathrm{i}}=\frac{\beta_{0}}{1+\exp \left[\left(\beta_{1}-\mathrm{I}_{\mathrm{i}}\right) / \beta_{2}\right]}+\varepsilon_{\mathrm{i}}
$$

Em que: $\overline{\mathrm{HDC}}_{\mathrm{i}}=$ altura média das árvores dominantes e codominantes da i-ésima unidade amostral (m); $\mathrm{I}_{\mathrm{i}}=$ idade da i-ésima unidade amostral (anos); $\exp =$ base do logaritmo neperiano; $\beta$ ' $s=$ parâmetros a serem estimados; $\varepsilon_{\mathrm{i}}=$ erro aleatório.

\section{Método da curva-guia para construção de curvas de índice de sítio}

Como exemplo, o modelo logístico de três parâmetros (Equação 1) será utilizado para estimativa da $\overline{\mathrm{HDC}}$ e construção das curvas de índice de sítio. Ressalta-se que o modelo adotado para tal procedimento deverá, de acordo com os critérios estatísticos, apresentar as melhores estatísticas de ajuste entre os modelos testados. 


$$
\overline{\mathrm{HDC}}_{\mathrm{i}}=\frac{\beta_{0}}{1+\exp \left[\left(\beta_{1}-\mathrm{I}_{\mathrm{i}}\right) / \beta_{2}\right]}+\varepsilon_{\mathrm{i}}
$$

Em que: $\overline{\mathrm{HDC}}_{\mathrm{i}}=$ altura média das árvores dominantes e codominantes da i-ésima unidade amostral (m); $\mathrm{I}_{\mathrm{i}}=$ idade da i-ésima unidade amostral (anos); exp = base do logaritmo neperiano; $\beta$ 's = parâmetros a serem estimados; $\varepsilon_{\mathrm{i}}=$ erro aleatório.

O método utilizado para construção das curvas de índice de sítio foi o da curva-guia. A idade de referência $\left(\mathrm{I}_{\text {ref }}\right)$ é de 7 anos, definida pela empresa da qual os dados foram coletados.

Quando $\mathrm{I}_{\mathrm{i}}=\mathrm{I}_{\text {ref }} \Rightarrow \overline{\mathrm{HDC}}_{\mathrm{i}}=\mathrm{S}$, Equação 2 .

$$
\overline{\mathrm{HDC}}_{2_{\mathrm{i}}}=\mathrm{S}_{\mathrm{i}}=\frac{\hat{\beta}_{0}}{1+\exp \left[\left(\hat{\beta}_{1}-\mathrm{I}_{\mathrm{ref}}\right) / \hat{\beta}_{2}\right]}
$$

Em que: $\overline{\mathrm{HDC}}_{\mathrm{i}}$ Isolando o parâmetro $\hat{\beta}_{0}$ (assíntota) do Modelo 1 e substituindo na Expressão 2, tem-se a estimativa da nas diferentes idades e índices de sítios, Equação 3.

$$
\overline{\mathrm{HDC}}_{2_{\mathrm{i}}}=\mathrm{S}_{\mathrm{i}}=\frac{\overline{\mathrm{HDC}}_{\mathrm{i}_{\mathrm{i}}} *\left\{1+\exp \left[\left(\hat{\beta}_{1}-\mathrm{I}_{\mathrm{i}}\right) / \hat{\beta}_{2}\right]\right\}}{1+\exp \left[\left(\hat{\beta}_{1}-\mathrm{I}_{\text {ref }}\right) / \hat{\beta}_{2}\right]}
$$

Em que: $\overline{\mathrm{HDC}}_{2_{\mathrm{i}}}=$ estimativa da altura média das árvores dominantes e codominantes na idade de referência da i-ésima unidade amostral $(\mathrm{m}) ; \mathrm{S}_{\mathrm{i}}=$ estimativa do índice de sítio da i-ésima unidade amostral; $\mathrm{I}_{\text {ref }}=$ idade de referência (7 anos).

A partir dos valores de $\overline{\mathrm{HDC}}_{2}$ estimados na idade de referência, e utilizando uma amplitude definida é possível definir classes de índice de sítio. Definidas as classes de índice de sítio, estima-se o parâmetro $\beta_{0_{j}}$ para cada classe de sítio, Equação 4.

$$
\beta_{0_{\mathrm{j}}}=\operatorname{classe}_{\mathrm{j}}\left\{1+\left[\exp \left(\hat{\beta}_{1}-\mathrm{I}_{\text {ref }}\right) / \hat{\beta}_{2}\right]\right\}
$$

Em que: $\beta_{0_{j}}=$ parâmetro estimado da j-ésima classe de sítio; classe ${ }_{j}=$ valor da $j$-ésima classe de sítio (m).

Estimados os valores de $\beta_{0_{j}}$ para cada classe de sítio é obtida a expressão que propiciará a estimativa da $\overline{\mathrm{HDC}}_{\mathrm{i}}$ para as várias idades vinculadas a qualquer classe de sítio, Equação 5. 


$$
\overline{\mathrm{HDC}}_{i_{\mathrm{j}}}=\frac{\hat{\beta}_{0_{\mathrm{j}}}}{1+\exp \left[\left(\hat{\beta}_{1}-\mathrm{I}_{\mathrm{i}}\right) / \hat{\beta}_{2}\right]}
$$

Em que: $\overline{\mathrm{HDC}}_{\mathrm{j}}=$ estimativa da altura média das árvores dominantes e codominantes da i-ésima unidade amostral vinculada a j-ésima classe de sítio.

\section{Avaliação da qualidade de ajuste dos modelos}

Os testes de hipótese "t" de Student e o p-value foram utilizados para inferir sobre a relevância das covariáveis, representadas pelas estimativas dos parâmetros, em relação à variável de interesse. Ao contrário do teste " $\mathrm{t}$ ", que compara o valor da estatística obtida da amostra com o valor da estatística do teste definido em função de um $\alpha$ fixado, a estatística $p$-value compara o valor $\mathrm{p}$ com o $\alpha$ fixado. Assim, a hipótese $\mathrm{H}_{0}$ é rejeitada, ou seja, $(\beta \neq 0)$, se o valor $\mathrm{p}$ for menor ou igual a $\alpha(5 \%)$. Isso significa que o " $\mathrm{t}$ " calculado está na região de rejeição de $\mathrm{H}_{0}$.

Para avaliação dos modelos e verificação das suposições básicas de análise de regressão foram utilizadas a análise gráfica dos resíduos e a análise do histograma de classes de resíduos, juntamente com algumas medidas de acurácia que serviram de subsídio na comparação dos modelos. Os modelos ajustados tiveram sua precisão avaliada pelas seguintes estatísticas:

a) Erro padrão residual absoluto e percentual

$$
\begin{gathered}
S_{y x}=\sqrt{\frac{\sum_{i=1}^{n}(Y-\hat{Y})^{2}}{n-p}} \\
S_{y x}(\%)=\frac{S_{y x}}{\bar{Y}} 100
\end{gathered}
$$

Em que: $\mathrm{S}_{\mathrm{yx}}=$ erro padrão residual absoluto $(\mathrm{m}) ; \mathrm{S}_{\mathrm{yx}}(\%)=$ erro padrão relativo (\%); $\mathrm{Y}=$ valor observado da variável dependente; $\hat{Y}=$ valor estimado da variável dependente; $\mathrm{n}=$ número de observações; $\mathrm{p}=$ número de parâmetros do modelo.

b) Critério de informação de Akaike (AIC) - Sakamoto, Ishiguro e Kitagawa (1986)

$$
\mathrm{AIC}=-2 \ln (\mathrm{mv})+2 \mathrm{p}
$$

Em que: $\mathrm{mv}=$ valor da máxima verossimilhança; $\ln =$ logaritmo neperiano.

c) Critério de informação Bayesiano (BIC) - Schwarz (1978) 


$$
\mathrm{BIC}=-2 \ln (\mathrm{mv})+\mathrm{p} \ln (\mathrm{n})
$$

d) Teste da razão da máxima verossimilhança (TRMV)

$$
\operatorname{TRMV}=2\left[\ln \left(\mathrm{mv}_{2}\right)-\ln \left(\mathrm{mv}_{1}\right)\right]
$$

Em relação aos critérios, sabe-se que, quanto menores os valores de AIC e BIC melhor o modelo ajustado, pois penalizam modelos com número excessivo de parâmetros, selecionando modelos mais parcimoniosos. O teste TRMV é uma ferramenta de comparação de modelos aninhados, dado pela diferença entre os valores máximos da função de verossimilhança dos modelos. Esse valor é testado pela estatística qui-quadrado $\left(x^{2}\right)$. Se o valor de TRMV for significativo em nível de $\alpha$ de probabilidade, o modelo com maior valor de máxima verossimilhança apresenta maior precisão.

A partir da análise das estatísticas, procederá a ordenação das funções segundo o maior grau de precisão $\left(\mathrm{S}_{\mathrm{yx}} \%\right)$. O melhor modelo hipsométrico e de sítio será utilizado para estimativa da altura total e da $\overline{\mathrm{HDC}}$ na idade de referência, respectivamente.

TABELA 5: Estatísticas de ajuste dos modelos de relação hipsométrica e seus respectivos parâmetros estimados.

TABLE 5: Fit statistics of the hypsometric relation models and their estimated parameters.

\begin{tabular}{|c|c|c|c|c|}
\hline Parâmetro & Estimativa & Erro Padrão & $\mathrm{t}$ & p-valor \\
\hline$\beta_{0}$ & 9,1166 & 1,159 & 7,869 & $<0,0001$ \\
\hline$\beta_{1}$ & 32,2504 & 0,385 & 83,653 & $<0,0001$ \\
\hline$\beta_{2}$ & 12,5550 & 0,347 & 36,207 & $<0,0001$ \\
\hline$\beta_{3}$ & 3,5418 & 0,259 & 13,664 & $<0,0001$ \\
\hline \multicolumn{5}{|c|}{ Logístico 3 Parâmetros $\left(\mathrm{S}_{\mathrm{yx}}=11,94 \%\right)$} \\
\hline$\beta_{0}$ & 33,84713 & 0,436 & 77,691 & $<0,0001$ \\
\hline$\beta_{1}$ & 10,00832 & 0,142 & 70,643 & $<0,0001$ \\
\hline$\beta_{2}$ & 5,11851 & 0,203 & 25,227 & $<0,0001$ \\
\hline \multicolumn{5}{|c|}{ Parabólico $\left(\mathrm{S}_{\mathrm{yx}}=12,03 \%\right)$} \\
\hline$\beta_{0}$ & $-4,243815$ & 0,823 & $-5,154$ & $<0,0001$ \\
\hline$\beta_{1}$ & 2,623149 & 0,099 & 26,362 & $<0,0001$ \\
\hline$\beta_{2}$ & $-0,046629$ & 0,003 & $-15,613$ & $<0,0001$ \\
\hline \multicolumn{5}{|c|}{ Curtis $\left(\mathrm{S}_{\mathrm{yx}}=12,38 \%\right)$} \\
\hline$\beta_{0}$ & 3,81222 & 0,011 & 352,540 & $<0,0001$ \\
\hline$\beta_{1}$ & $-9,13920$ & 0,160 & $-57,030$ & $<0,0001$ \\
\hline
\end{tabular}

Logístico 4 Parâmetros $\left(\mathrm{S}_{\mathrm{yx}}=11,89 \%\right)$ 
TABELA 6: Critério de informação de Akaike (AIC) e critério de informação Bayesiano (BIC) para os modelos hipsométricos ajustados.

TABLE 6: Akaike information criterion and Bayesian information criterion for the adjusted hypsometric models.

\begin{tabular}{lcc}
\hline \multicolumn{1}{r}{ Modelo } & AIC & BIC \\
\hline Logístico 4 Parâmetros & 10889,240 & 10917,590 \\
Logístico 3 Parâmetros & 10907,630 & 10930,310 \\
Parabólico & 10936,870 & 10959,560 \\
Curtis & 11452,730 & 11469,740 \\
\hline
\end{tabular}

\section{RESULTADOS E DISCUSSÕES}

\section{Análise dos modelos hipsométricos}

Após o ajuste dos modelos hipsométricos obtiveram-se as estatísticas que possibilitaram a ordenação das funções, conforme Tabela 5.

De acordo com o teste " $t$ ", nota-se que todos os parâmetros são significativos $(\mathrm{p}<0,05)$. Considerando como critério de seleção as estatísticas $\mathrm{S}_{\mathrm{yx}}(\%)$, AIC e BIC, Tabela 5 e 6 , nota-se que os modelos não lineares obtiveram vantagem em relação aos modelos lineares. O modelo logístico de quatro parâmetros obteve as melhores estatísticas de precisão, seguido pelo modelo logístico de três parâmetros, o modelo parabólico e o modelo de Curtis, respectivamente. Na Tabela 6 são apresentadas as estatísticas dos modelos ajustados.

Com o intuito de verificar se as suposições da análise de regressão são atendidas, utilizou-se o gráfico de análise residual, representado pela Figura 1 (a), e o gráfico representando a relação entre a altura total estimada e a altura total observada, conforme a Figura 1 (b). Estas análises são demonstradas apenas



(a)

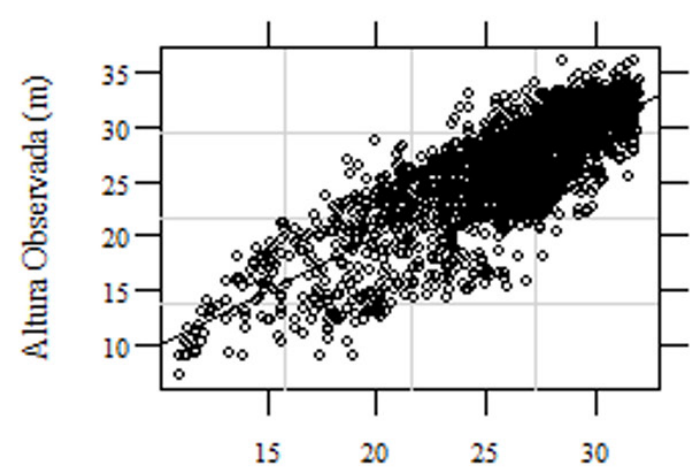

Altura Estimada (m)

(b)

FIGURA 1: (a) Gráfico de resíduo; (b) Relação entre altura total estimada (m) e altura total observada (m), para o modelo logístico clássico de quatro parâmetros.

FIGURE 1: (a) Graph of residue; (b) Relationship between estimated total height (m) and total height observed (m), for the classic four parameters logistic model. 


$$
\mathrm{H}_{\mathrm{t}_{\mathrm{i}}}=\beta_{0}+\frac{\beta_{1}-\beta_{0}}{1+\exp \left[\left(\beta_{2}-\mathrm{DAP}_{\mathrm{i}}\right) / \beta_{3}\right]}+\varepsilon_{\mathrm{i}}
$$

Em que: $\beta_{0}=\beta_{00}+\beta_{01} \mathrm{I}+\beta_{02} \mathrm{~S}+\beta_{03} \mathrm{G} ; \beta_{1}=\beta_{10}+\beta_{11} \mathrm{I}+\beta_{12} \mathrm{~S} ; \beta_{2}=\beta_{20}+\beta_{21} \mathrm{I}+\beta_{22} \mathrm{~S}+\beta_{23} \mathrm{G} ; \beta_{3}=\beta_{30}+\beta_{31} \mathrm{I}+\beta_{32} \mathrm{~S}+\beta_{33}$ $\mathrm{G} ; \mathrm{I}=$ idade do povoamento (anos); $\mathrm{S}=$ índice de sítio $(\mathrm{m}) ; \mathrm{G}=$ área basal por hectare $\left(\mathrm{m}^{2} \mathrm{ha}^{-1}\right)$.

A Tabela 7 contém as estatísticas de ajuste do modelo logístico de quatro parâmetros com inclusão de covariáveis. Pode-se observar que todos os parâmetros foram significativos em nível de $5 \%$ de significância, com exceção do intercepto, representado pelo parâmetro $\beta_{10}$, provavelmente devido ao fato de o parâmetro $\beta_{10}$ perder força pela adição das covariáveis (idade, sítio e área basal) ao modelo. Nesse caso, esse fato deve ser desprezível quando se tratar da não significância de algum intercepto, pois tais variáveis influenciam a relação altura-diâmetro (MENDONÇA et al., 2011).

A Tabela 8 mostra as estatísticas de comparação entre o modelo logístico original de quatro parâmetros (clássico) e o modelo com adição de covariáveis. Avaliando os resultados, observa-se que o parâmetro TRMV foi significativo, comprovando uma melhora do modelo com inclusão de covariáveis em relação ao modelo original. Tal fato é comprovado pela melhoria significativa em todos os parâmetros estatísticos avaliados.

As Figuras 2 (a), 2 (b) e 2 (c) mostram a análise residual, a relação altura total estimada versus altura total observada e as observações agrupadas em classes de resíduos, respectivamente, para o modelo logístico de quatro parâmetros com adição de covariáveis.

TABELA 7: Estatísticas de ajuste do modelo logístico de quatro parâmetros com adição de covariáveis. TABLE 7: Statistics of the adjust of logistic model of four parameters with adding of covariates.

\begin{tabular}{lccccc}
\hline \multicolumn{5}{c}{ Logístico 4 Parâmetros com Adição de Covariáveis $\left(\mathrm{S}_{\mathrm{yx}}=6,44 \%\right)$} \\
\hline \multicolumn{1}{c}{ Parâmetro } & $\begin{array}{c}\text { Covariante } \\
\text { Associada }\end{array}$ & Estimativa & Erro Padrão & $\mathrm{t}$ & $\mathrm{p}$-valor \\
\hline$\beta_{00}$ & Intercepto & $-71,64253$ & 15,544 & $-4,609$ & $<0,001$ \\
$\beta_{01}$ & Idade & 11,65198 & 1,466 & 7,947 & $<0,001$ \\
$\beta_{02}$ & $\mathrm{~S}$ & $-1,25026$ & 0,350 & $-3,572$ & $<0,001$ \\
$\beta_{03}$ & $\mathrm{G}$ & 2,00919 & 0,230 & 8,714 & $<0,001$ \\
$\beta_{10}$ & Intercepto & $-4,01248$ & 2,226 & $-1,802$ & 0,072 \\
$\beta_{11}$ & Idade & 1,70009 & 0,171 & 9,920 & $<0,001$ \\
$\beta_{12}$ & $\mathrm{~S}$ & 0,73627 & 0,061 & 11,965 & $<0,001$ \\
$\beta_{20}$ & Intercepto & $-18,72199$ & 3,944 & $-4,747$ & $<0,001$ \\
$\beta_{21}$ & $\mathrm{Idade}$ & 3,83527 & 0,359 & 10,666 & $<0,001$ \\
$\beta_{22}$ & $\mathrm{~S}$ & $-0,29152$ & 0,098 & $-2,969$ & $<0,001$ \\
$\beta_{23}$ & $\mathrm{G}$ & 0,62048 & 0,063 & 9,838 & $<0,001$ \\
$\beta_{30}$ & Intercepto & 6,44956 & 1,986 & 3,247 & $<0,001$ \\
$\beta_{31}$ & Idade & $-1,24937$ & 0,162 & $-7,716$ & $<0,001$ \\
$\beta_{32}$ & $\mathrm{~S}$ & 0,32756 & 0,053 & 6,181 & $<0,001$ \\
$\beta_{33}$ & $\mathrm{G}$ & $-0,28636$ & 0,021 & $-13,843$ & $<0,001$ \\
\hline
\end{tabular}

Em que: $\mathrm{S}=$ índice de sítio $(\mathrm{m}) ; \mathrm{G}=$ área basal por hectare $\left(\mathrm{m}^{2}\right.$ ha $\left.\mathrm{a}^{-1}\right)$.

TABELA 8: Estatísticas de comparação entre o modelo logístico original com quatro parâmetros e o modelo logístico com adição de covariáveis.

TABLE 8: Statistics comparison between the original logistic model, with four parameters, and the logistic model adding covariates.

\begin{tabular}{llcccc}
\hline \multicolumn{1}{c}{ Modelo } & G.L. & AIC & BIC & TRMV & p-valor \\
\hline Original & 2141 & 10889,240 & 10917,590 & & $<0,0001$ \\
Covariáveis & 2130 & 8286,576 & 8377,311 & 2624,66 & \\
\hline
\end{tabular}

Em que: $\mathrm{AIC}=$ critério de informação de Akaike, $\mathrm{BIC}=$ critério de informação Bayesiano; TRMV = teste de razão da máxima verossimilhança. 
para o modelo logístico com quatro parâmetros, que apresentou as melhores estatísticas de ajuste.

Na Figura 1 (a), observa-se uma boa distribuição dos resíduos para o modelo logístico clássico de quatro parâmetros, atendendo à pressuposição de independência dos resíduos. O mesmo não pode ser afirmado para a pressuposição de homocedasticidade dos resíduos, ou seja, o erro apresentou uma leve variância não constante. Nesse caso, pelo fato de o erro sofrer uma leve variação do ideal, a inferência pode não ser comprometida, ou seja, este modelo pode ser usado para a relação hipsométrica, sem medo de que as estimativas sejam tendenciosas.

Pode-se concluir após análise da Figura 1 (b), que, apesar dos valores estimados estarem um pouco distantes dos valores observados, o modelo clássico conseguiu representar a variação real da relação hipsométrica. Esta relação pode ser melhorada com a inclusão de covariáveis.

Com o objetivo de obtenção de melhor precisão nas estimativas da altura total, o modelo logístico de quatro parâmetros foi decomposto adicionando ao modelo variáveis que explicam a variabilidade da altura. Com a inclusão de covariáveis, o modelo logístico de quatro parâmetros foi ajustado conforme estrutura apresentada pelo Modelo 11.

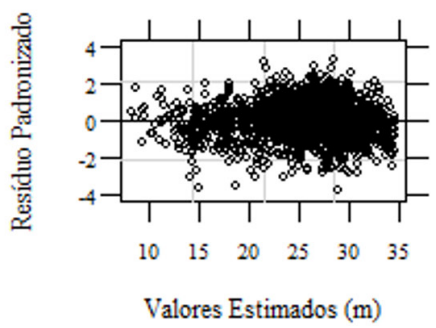

(a)

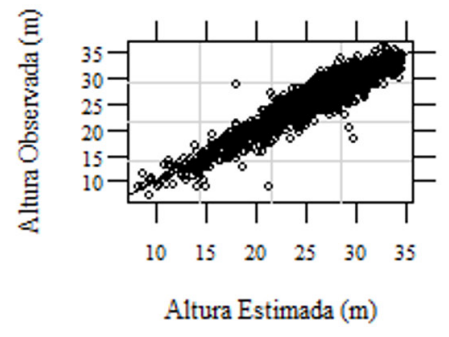

(b)

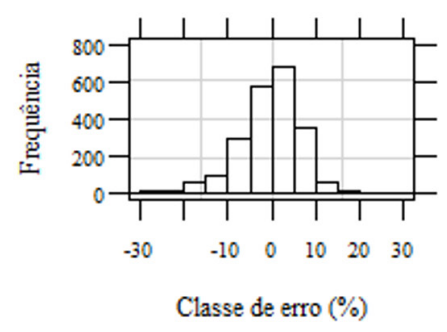

(c)

FIGURA 2: (a) Gráfico de resíduo; (b) Relação entre altura total estimada (m) e altura total observada (m); (c) Histograma de classes de resíduos (\%), para o modelo logístico de quatro parâmetros com adição de covariáveis.

FIGURE 2: (a) Graph of residue; (b) Relationship between estimated total height (m) and observed total height $(\mathrm{m}),(\mathrm{c})$ histogram of residue classes $(\%)$ to the four parameters logistic model adding covariates.

Nota-se que todas as pressuposições da análise de regressão foram atendidas, inclusive a pressuposição de homocedasticidade. A pressuposição de normalidade é verificada pelo histograma de classes de resíduos na Figura 2 (c). Estas melhorias, tanto na análise residual quanto na relação entre altura estimada e observada, se devem à inclusão de covariáveis ao modelo.

Verifica-se na Figura 2 (b) uma melhoria significativa em comparação com a Figura 1 (b), na qual nota-se uma boa qualidade no ajuste do modelo. Este fato é comprovado pela proximidade dos valores estimados em relação aos valores observados com a média. Estes resultados corroboram todas as estatísticas analisadas, quando comparados com as estatísticas do modelo clássico. Resultados semelhantes foram encontrados nos trabalhos de Guimarães et al. (2009) e Mendonça et al. (2011) com a adição de covariáveis.

\section{Análise dos modelos para estimativa da altura média das árvores dominantes e codominantes}

Na Tabela 9 podem ser observadas as estatísticas de ajuste dos modelos utilizados para estimativa da altura média das árvores dominantes e codominantes. Nota-se que todos os parâmetros são significativos $(\mathrm{p}<0,05)$. Considerando apenas a significância dos parâmetros, chega-se à conclusão de que todos os modelos podem ser utilizados para estimativa da $\overline{\mathrm{HDC}}$. Com relação ao $\mathrm{S}_{\mathrm{yx}}(\%)$, observa-se que o modelo logístico obteve o melhor resultado (5,64 \%), seguido pelos modelos de Bailey e Clutter (5,68 \%), Chapman 
TABELA 9: Estatísticas de ajuste dos modelos para classificação da capacidade produtiva e seus respectivos parâmetros estimados.

TABLE 9: Fit statistics of models for the classification of productive capacity and their estimated parameters.

\begin{tabular}{ccccc}
\hline \multicolumn{5}{c}{ Logístico $\left(\mathrm{S}_{\mathrm{yx}}=5,64 \%\right)$} \\
\hline Parâmetro & Estimativa & Erro Padrão & t-valor & p-valor \\
\hline$\beta_{0}$ & 33,72575 & 0,630 & 53,535 & $<0,0001$ \\
$\beta_{1}$ & 2,29369 & 0,071 & 32,086 & $<0,0001$ \\
$\beta_{2}$ & 1,51997 & 0,122 & 12,447 & $<0,0001$ \\
\hline$\beta_{0}$ & Bailey e Clutter $\left(\mathrm{S}_{\mathrm{yx}}=5,68 \%\right)$ & $<0,0001$ \\
$\beta_{1}$ & 1,524 & 23,390 & $<0,0001$ \\
$\beta_{2}$ & $-0,23891$ & 0,017 & $-14,140$ & $<0,0001$ \\
\hline$\beta_{0}$ & 1,19547 & 0,103 & 11,640 & $<0,0001$ \\
$\beta_{1}$ & Chapman e Richards $\left(\mathrm{S}_{\mathrm{yx}}=5,68 \%\right)$ & $<0,0001$ \\
$\beta_{2}$ & 1,461 & 24,977 & $<0,0001$ \\
\hline$\beta_{0}$ & $-0,35877$ & 0,061 & $-5,918$ & $<0,0001$ \\
$\beta_{1}$ & 1,32285 & 0,212 & 6,233 & $<0,0001$ \\
\hline
\end{tabular}

TABELA 10: Critérios estatísticos para os modelos de sítio ajustados.

TABLE 10: Statistical criteria for the adjusted site models.

\begin{tabular}{lcc}
\hline \multicolumn{1}{c}{ Modelo } & AIC & BIC \\
\hline Logístico & 547,450 & 559,357 \\
Bailey e Clutter & 549,431 & 561,338 \\
Chapman e Richards & 549,769 & 561,676 \\
Schumacher & 553,406 & 562,336 \\
\hline
\end{tabular}

e Richards (5,68 \%), e Schumacher (5,78 \%).

Observados o AIC e o BIC presentes na Tabela 10, nota-se que o modelo logístico foi superior para todos os critérios analisados, seguido pelos modelos de Bailey e Clutter, Chapman e Richards, e pelo modelo de Schumacher, respectivamente. Os resultados apresentados na Tabela 10 corroboram a estatística do $\mathrm{S}_{\mathrm{yx}}(\%)$, apresentada na Tabela 9.

\section{Estimativa da $\overline{\mathrm{HDC}}$ e construção de curvas de índice de sítio}

De acordo com os critérios estatísticos, sabe-se que o modelo logístico (Equação 1) apresentou as melhores estatísticas em comparação com os modelos analisados, sendo esse modelo utilizado para estimativa da altura média das árvores dominantes e codominantes, e construção das curvas de índice de sítio conforme metodologia apresentada no item Método da curva-guia para construção de curvas de índice de sítio.

A amplitude adotada para a confecção de curvas de sítio foi de quatro metros. Na Tabela 11 são apresentados os limites das classes de sítio. 


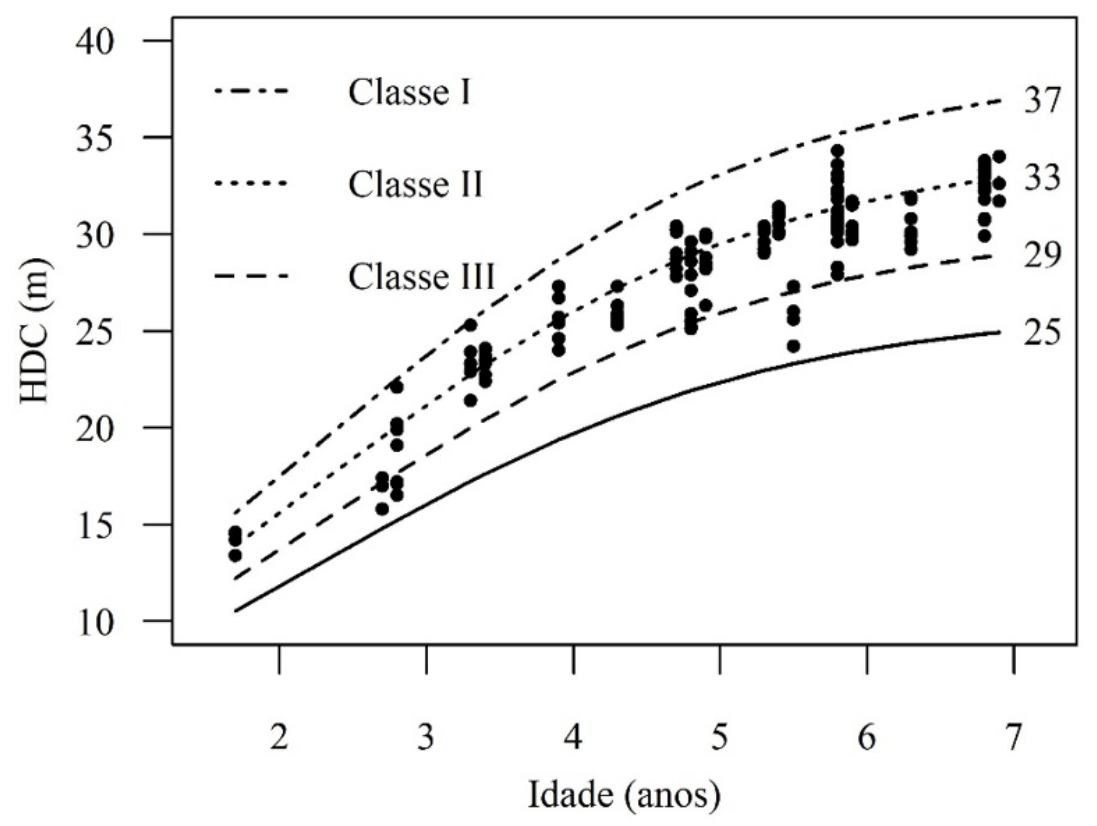

FIGURA 3: Curvas de índice de sítio para clones de eucalipto na empresa Anglo American.

FIGURE 3: Site index curves for eucalypt clones at Anglo American company.

TABELA 11: Limite das classes de sítio para o modelo logístico.

TABLE 11: Limit of site classes for the logistic model.

\begin{tabular}{ccc}
\hline Classe & Limite inferior $(\mathrm{m})$ & Limite superior $(\mathrm{m})$ \\
\hline I & 33,1 & 37 \\
II & 29,1 & 33 \\
III & 25 & 29 \\
\hline
\end{tabular}

Após a definição das classes de sítio foram plotadas as curvas de índice de sítio (Figura 3). Nota-se que a altura média das árvores dominantes e codominantes estão, em maior frequência, nos sítios I e II.

\section{CONCLUSÕES}

A utilização de modelos não lineares para estimativa da relação hipsométrica e classificação de sítio mostrou-se superior em todas as estatísticas analisadas. Estes modelos são preferíveis aos modelos lineares, pois produzem estimativas de alta precisão, são parcimoniosos, seus parâmetros são interpretáveis, além de sua ampla aplicabilidade em qualquer base de dados de origem florestal.

O modelo logístico de quatro parâmetros foi o mais preciso para estimar a relação hipsométrica, enquanto que o logístico de três parâmetros, o melhor para estimativa do crescimento em altura média das árvores dominantes e codominantes para clones de eucalipto.

A utilização da técnica de inclusão de covariáveis melhorou significativamente a precisão na estimativa da relação hipsométrica, destacando sua importância e potencial na utilização em estudos de crescimento e produção. 


\section{AGRADECIMENTOS}

À CAPES (Coordenação de Aperfeiçoamento de Pessoal de Ensino Superior) pelo apoio financeiro e à empresa Anglo American Fosfato Brasil Ltda., pela disponibilização dos dados para realização do estudo.

\section{REFERÊNCIAS}

AMATEIS, R. L.; BURKHART, H. E. Site index curves for loblolly pine plantations on cutover site-prepared lands. Southern Journal of Applied Forestry, Washington, v. 9, p. 166-169, 1985.

BARTOSZECK, A. C. D. P. E. S. et al. Dinâmica da relação hipsométrica em função da idade, do sítio e da densidade inicial de povoamentos de bracatinga da Região Metropolitana de Curitiba, PR. Revista Árvore, Viçosa, MG, v. 28, n. 4, p. 517-533, jul./ago. 2004a.

BARTOSZECK, A. C. D. P. E. S. et al. Modelagem da relação hipsométrica para bracatingais da região metropolitana de Curitiba PR. Floresta, Curitiba, v. 32, n. 2, p. 189-204, 2004 b.

CALEGARIO, N. et al. Melhoria da qualidade de ajuste de modelos biométricos florestais pelo emprego da teoria dos modelos não lineares generalizados. Scientia Forestalis, Piracicaba, n. 69, p. 38-50, 2005.

CAMPOS, J. C. C.; LEITE, H. G. Mensuração florestal: perguntas e respostas. 3. ed. Viçosa, MG: UFV, 2009. $548 \mathrm{p}$.

CORRAL RIVAS, J. J. et al. Compatible height and site index models for five pine species in El Salto, Durango, Mexico. Forest Ecology and Management, Amsterdam, v. 201, n. 2/3, p. 145-160, 2004.

DIÉGUEZ-ARANDA, U.; BURKHART, H. E.; RODRÍGUEZ-SOALLEIRO, R. Modeling dominant height growth of radiata pine (Pinusradiata D. Don) plantations in north-western Spain. Forest Ecology and Management, Amsterdam, v. 215, n. 1/3, p. 271-284, 2005.

GUIMARÃES, M. A. M. et al. Height-diameter models in forestry with inclusion of covariates. Cerne, Lavras, v. 15, n. 3, p. 313-321, jul./set. 2009.

KITIKIDOU, K.; PETROU, P.; MILIOS, E. Dominant height growth and site index curves for calabrian pine (Pinusbrutia Ten.) in central Cyprus. Renewable and Sustainable Energy Reviews, New York, v. 16, n. 2, p. 1323-1329, 2012.

MACHADO, S. D. A. et al. Análise do comportamento da relação hipsométrica com respeito à idade para plantações de Pinus elliotti no estado do Paraná. Cerne, Lavras, v. 1, n. 1, p. 5-12, 1994.

MENDONÇA, A. R. et al. Modelos hipsométricos e de crescimento em altura das árvores dominantes e codominantes para Pinus caribaea var. hondurensis. Scientia Forestalis, Piracicaba, v. 39, n. 90, p. 151-160, jun. 2011.

PINHEIRO, J. C.; BATES, D. M. Mixed-Effects Models in S and S-Plus. New York: Springer-Verlag, 2000. $528 \mathrm{p}$.

SAKAMOTO, Y.; ISHIGURO, M.; KITAGAWA, G. Akaike information criterion statistics. Dordrecht: Kluwer Academic, 1986. 256 p.

SCHWARZ, P. H. Estimating the dimension of a model. Annals of Statistics, California, v. 6, p. 461-464, 1978.

TEMESGEN, H.; GADOW, K. Generalized height-diameter models-an application for major tree species in complex stands of interior British Columbia. European Journal of Forest Research, Georgetown, v. 123, n. 1, p. 45-51, 2004.

TOMÉ, M.; RIBEIRO, F.; FAIAS, S. Relação hipsométrica geral para Eucalyptus globulus Labill. em Portugal. Silva Lusitana, Oeiras, v. 15, n. 1, p. 41-55, 2007.

TONINI, H.; SCHNEIDER, P. R.; FINGER, C. A. G. Curvas de índice de sítio para povoamentos clonais de Eucalyptus saligna Smith para a depressão central e serra do Sudeste, Rio Grande do Sul. Ciência Florestal, Santa Maria, v. 16, n. 1, p. 27-43, 2006. 